\title{
Senecio guatulamensis, nueva especie de Asteraceae endémica de Chile
}

\section{Senecio guatulamensis, a new Chilean endemic Asteraceae species}

\author{
Mélica Muñoz-SchicK*, Andrés Moreira-Muñoz ${ }^{2}$ \& Alain de Trenqualye ${ }^{3}$ \\ ${ }^{1}$ Museo Nacional de Historia Natural. Interior Quinta Normal s/n, Casilla 787, Santiago, Chile. \\ ${ }^{2}$ Instituto de Geografía, Pontificia Universidad Católica de Valparaíso, Avenida Brasil 2241, Valparaíso, Chile. \\ ${ }^{3}$ Av. Luis Pasteur 6006, Depto. 302, Santiago, Chile. \\ *melica4@gmail.com
}

\begin{abstract}
A new species of Senecio is described, on the basis of recent prospections in the Andes of Limarí valley, Coquimbo Region. The new species pertains to the Senecio section, series Andina. Differences with allied taxa are discussed.
\end{abstract}

El género Senecio es cosmopolita y uno de los más diversos de las angiospermas; por su variabilidad, su clasificación taxonómica a nivel de especie ha sido problemática, existiendo gran cantidad de sinónimos. Una delimitación amplia del género reconoce cerca de 3000 especies (Vincent 1996), y una más estricta las acerca a 1200 especies (Freire et al. 2014). Las especies se encuentran distribuidas en todo el mundo, a excepción de las islas del Pacífico y del continente Antártico. La mayor concentración de especies se encuentra en América, Asia y África (Freire et al. 2014).

En relación con su forma de vida, pueden ser hierbas anuales o perennes, subarbustos, arbustos o árboles pequeños. Entre los más notables se encuentran los Senecio gigantes de África Oriental. Las especies arborescentes del género Robinsonia, endémico del Archipiélago Juan Fernández, han sido propuestas para su inclusión dentro del género Senecio (Pelser et al. 2007, 2010). Algunas especies son cosmopolitas como S. vulgaris L., nativa de Europa, Asia occidental y norte de África; en Chile se encuentra desde Antofagasta a Magallanes. Otras especies constituyen malezas ampliamente distribuidas, como por ejemplo $S$. aquaticus Hill subsp. barbareifolius (Wimm. et Grab.) Walters originario de Europa y muy común en Chile desde la Región del Biobío a la de Los Lagos.

En Chile, Senecio constituye con creces el género más diverso entre las plantas vasculares, con alrededor de 224 especies (Teillier \& Marticorena 2006). El nivel de endemismo del género en Chile es muy alto, con 103 especies (46\%) (Moreira-Muñoz 2011).

Las especies con distribuciones geográficas restringidas, son importantes en el marco de la conservación de la biodiversidad de Chile, particularmente en el Norte Chico y en la Zona Central, toda vez que su hábitat suele estar muy intervenido por el sobrepastoreo y por la expansión de la frontera agrícola. Al respecto, si bien una buena parte de las especies de Senecio se distribuye en varias regiones, no pocas ocupan un rango geográfico muy restringido; algunos ejemplos son Senecio garaventai Cabrera, conocido sólo del cerro La Campana, Región de Valparaíso, S. benaventianus J. Rémy, desde las provincias del Limarí a la del Choapa, Región de Coquimbo y S. jilesii Cabrera, de la precordillera del Elqui y del Limarí, Región de Coquimbo (Cabrera 1949, Arancio et al. 2001).

Prospecciones recientes en el marco del proyecto "Geografía del Endemismo" (Fondecyt 1150425) han permitido confirmar la presencia de algunas especies crípticas o escasamente recolectadas en Chile, revisar el rango de distribución de las especies en el gradiente de latitud y altitud y el descubrimiento de la especie que se describe a continuación.

Senecio guatulamensis Muñoz-Schick, Moreira \& Trenqualye, sp. nov.

TIPO: Chile, Región de Coquimbo, provincia de Limarí, Guatulame, quebrada de La Coipa, 3051'36,9”' S 7057'22.9' W, 774 msnm, M. Muñoz n 5404, 3-X-2014 (Holotipo SGO, Isotipo CONC).

Subshrub entirely white lanate, leaves sessile, entire to dentate, capitula radiate, solitary or 2-3, almost sessile, achenes shortly white pilose on the ribs.

Subarbusto con un leve aroma agradable; ramificado, de 20-30 cm de alto; forma matas de 20-30 cm de diámetro. Planta densamente blanco lanosa, donde la lanosidad se va perdieron con la madurez en las hojas inferiores; tallos con 
entrenudos de 2-4 mm; hojas alternas, sésiles, lanceoladas, de 10-25 (-30) x 3-9 mm, algo abrazadoras en la base, variables, desde casi enteras hasta 7-dentadas en el margen, que es algo revoluto. Capítulos radiados, solitarios, a veces 2-3 muy cercanos entre sí, casi sésiles en el ápice de las ramas hojosas. Involucro acampanado de $12 \mathrm{~mm}$ de alto por 12-15 mm de diámetro, con 18-20 brácteas lanceoladas, agudas en el ápice, calículo de 7-8 brácteas lineares con una leve puntita oscura. Flores marginales 13-14-liguladas, lígula de 6-7 x 3-4 mm, color amarillo-oro; las centrales numerosas, tubulosas, de 9-10 $\mathrm{mm}$ de largo, amarillas, pentadentadas en el ápice, dientes triangulares, sobresalen del involucro, los estambres y los estigmas sobrepasan al tubo. Aquenios de 3,5-4 mm de largo, costados, cortamente blanco-pilosos en las costillas, papus de color crema, de 8 mm de largo (Fig. 1).
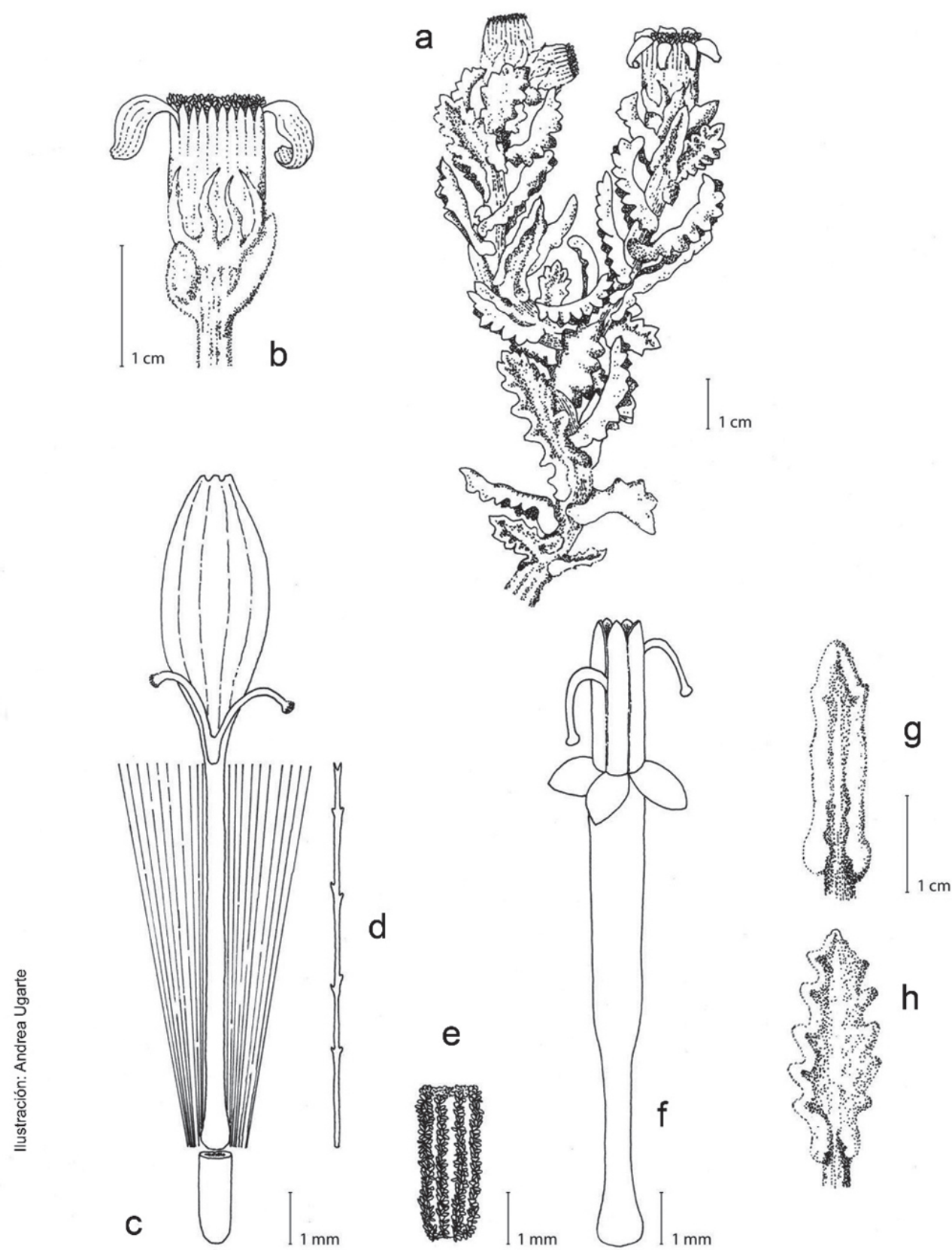

FIGURA 1. Senecio guatulamensis. a. hábito; b. capítulo; c. flor ligulada; d. detalle de un pelo del papus; e. aquenio; f. flor central; g. hoja casi entera, por el envés; h. hoja dentada, por el envés. Basados en el ejemplar Tipo, Muñoz n 5404.

Figure 1. Senecio guatulamensis. a. habit; b. capitulum; c. ligulate flower; d. pappus hair detail; e. achene; f. central flower; g. leaf almost entire, dorsal surface; h. dentate leaf, dorsal surface. Based on Type specimen, Muñoz n 5404. 

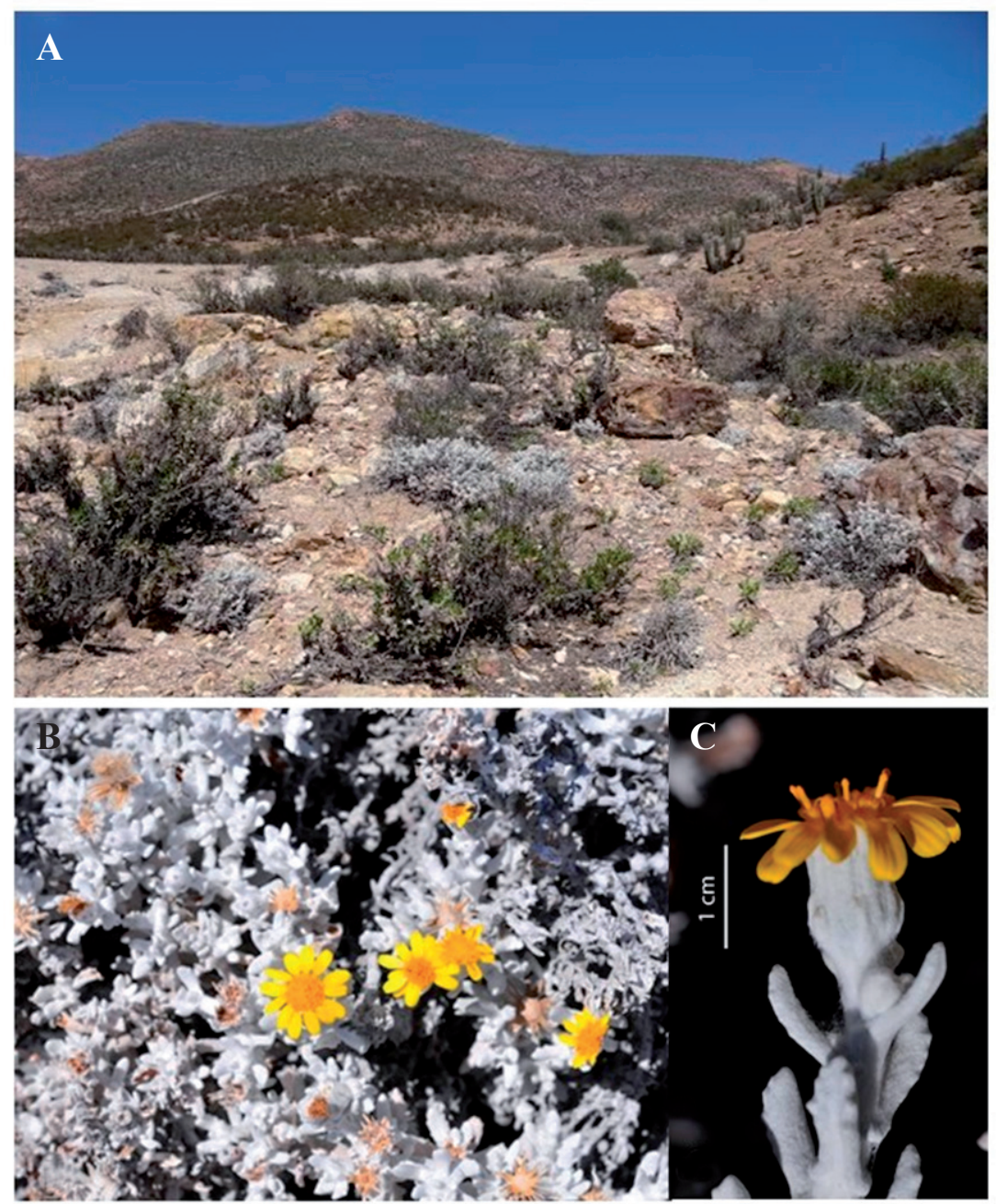

Figura 2. A. Hábitat de la especie B. Planta con flores. C. Detalle de ramita con capítulo (Fotos A. Nassella Muñoz, B-C. Sergio Moreira).

Figure 2. A. Species habitat; B. Plant with the flowers; C. Detail of stem with capitulum (Photos A. Nassella Muñoz, B-C. Sergio Moreira).

Especie endémica de la precordillera de Ovalle. Se la encontró en una explanada con pequeñas rocas por donde escurrió agua que probablemente bajaba desde los Baños de Guatulame, que se encuentran más arriba. En septiembre de 2008 se la observó en flor; en octubre de 2014 se la recolectó con flores y frutos.

OBSERVACIONES

Se observó una población de no más de 50 individuos. Otras especies que crecen en el área son Cistanthe arenaria (Cham.) Carolin ex Hershk., Dodonaea viscosa (L.) Jacq., una variedad de Eriosyce curvispina (Bertero ex Colla) Katt. y Haplopappus bezanillanus (J. Rémy) Reiche.

Por la combinación del hábito de subarbusto con los tallos hojosos hasta el ápice, las hojas lanosas, los capítulos solitarios o agrupados de 2-3 y radiados, esta nueva especie se debe agrupar con las de la sección Senecio, serie Andina
(Freire et al. 2014). Comparte hábito, algo de aroma y la lanosidad de las hojas con S. eriophyton J. Rémy, de la misma serie, pero se separa porque las hojas de este último son dentado-crespas, con dientes muy agudos, los capítulos tienen las lígulas menores, de hasta $4,5 \mathrm{~mm}$ de largo y los aquenios son glabros. Su distribución es en cordilleras de las regiones de Antofagasta a Coquimbo en Chile y en la Argentina (La Rioja a Mendoza).

Por la lanosidad y el hábito tiene cierta semejanza también con S. volckmannii Phil. de la sección Senecio, serie Chilensis, subserie Candidi (Freire et al. 2014), pero se separa porque este último tiene los capítulos discoideos y las hojas enteras. Esta especie vive en las altas cordilleras de Coquimbo en Chile y de Catamarca a Mendoza en la Argentina.

Se comparó a su vez con los Senecio descritos para la Flora de San Juan, Argentina (Kiesling 2013) por ser la 
Región de Coquimbo una región limítrofe con dicho país, pero no se encontró semejanza con ninguno de los de su grupo, excepto con $S$. eriophyton, situación que ya se comentó anteriormente.

La distribución conocida de $S$. guatulamensis está restringida a Guatulame, quebrada La Coipa, en la cordillera de Ovalle; es necesario realizar más prospecciones en la zona para determinar de manera más exacta su rango de distribución y su posible inclusión en alguna categoría de conservación (Fig. 2).

\section{AGRADECIMIENTOS}

Se agradece a Sergio Moreira E. y Nassella Muñoz Schick por acompañarnos en terreno y por sus magníficas fotos. A Andrea Ugarte, por la hermosa ilustración de los detalles de la especie. Proyecto Fondecyt 1150425.

\section{BIBLIOGRAFÍA}

Arancio, G., M. MuÑoz-Schick \& F.A. SQueo. 2001. Descripción de algunas especies con problemas de conservación en la IV Región de Coquimbo, Chile. En: F.A. Squeo, G. Arancio \& J.R.Gutiérrez (eds.), Libro Rojo de la flora nativa y de los Sitios Prioritarios para su conservación, Región de Coquimbo, pp. 63-103. Ediciones Universidad de La Serena, La Serena, Chile.

Cabrera, A.L. 1949. El género Senecio en Chile. Lilloa 15: 27-501.

Freire, S.E., L. Ariza Espinar, L. Salomón \& M.P. Hernández. 2014. Senecio L. En: F.O. Zuloaga, M.J. Belgrano \& A.M. Anton (eds.), Flora Vascular de la República Argentina, Vol. 7 (3), pp. 27-220. Estudio Sigma S.R.L., Buenos Aires, Argentina.

Kiesling, R. 2013. Flora de San Juan. Vol. 3-b, Zeta Editores, Mendoza. 338 pp.

Moreira-MuÑoz, A. 2011. Plant Geography of Chile. Series Plant and Vegetation, vol. 5, Springer, Dordrecht. 343 pp.

Pelser, P.B., B. Nordenstam, J.W. Kadereit \& L.E. Watson. 2007. An ITS phylogeny of tribe Senecioneae (Asteraceae) and a new delimitation of Senecio L. Taxon 56: 1077-1104.

Pelser, P.B., E.J. Tepe, A.H. Kennedy \& L.E. Watson. 2010. The fate of Robinsonia (Asteraceae): sunk in Senecio, but still monophyletic? Phytotaxa 5: 31-46.

Teillier, S. \& A. Marticorena. 2006. El género Senecio (Asteraceae): el más diverso de Chile. Revista Chagual (Jardín Botánico de Santiago) 4: 39-48.

VinCENT, P.L.D. 1996. Progress on clarifying the generic concept of Senecio based on an extensive world-wide sample of taxa. In: D.J.N. Hind \& H.J. Beentje (eds.), Compositae: Systematics. Proceedings of the International Compositae Conference, Vol. 1, pp. 597-611. Royal Botanic Gardens, Kew, Richmond, UK.

Recibido: 14.04.15

Aceptado: 08.03.16 